\title{
Febrile illness in a toddler: The diagnostic clue lays skin deep
}

\author{
Entesar Husain MD FRCPC ${ }^{1}$, David Cabral MD FRCPC ${ }^{2}$
}

\begin{abstract}
A five-year-old boy arrived in Canada fron China. The following day he developed a left cervical swelling, and was started on oral penicillin at a walk-in clinic. Subsequently, he developed fever and neck pain. Within $48 \mathrm{~h}$, the left side of his neck was swollen, erythematous and extremely painful. There was no history of cough, weight loss or night sweats. His past history was unremarkable, and all his immunizations were up-to-date. He had received the bacillus Calmette-Guérin (BCG) vaccine in the newborn period as part of the routine vaccination schedule in China. He was the only child of a Chinese couple who had just immigrated to Canada.

On examination, the boy was febrile $\left(38.5^{\circ} \mathrm{C}\right)$ and appeared unhappy. His eyes showed bilateral conjunctival erythema. He had leftsided torticollis. The left submandibular lymph node was tender and measured $3 \times 3 \mathrm{~cm}$. The overlying skin showed intense erythema. There was no other lymphadenopathy. The ear, nose and throat examination was normal. The rest of the systemic examination was also normal; in particular, there was no hepatosplenomegaly.

He was admitted to hospital and was started on parenteral antibiotics: clindamycin and cefurxime. Investigations revealed a total leukocyte count of $21.12 \times 10^{9} / \mathrm{L}$ : absolute neutrophils $16.5 \times 10^{9} / \mathrm{L}$, bands $1.6 \times 10^{9} / \mathrm{L}$ ); and lymphocytes $1.27 \times 10^{9} / \mathrm{L}$ ). The platelet count was $381 \times 10^{9} / \mathrm{L}$, hemoglobin $108 \mathrm{~g} / \mathrm{L}$ and sedimentation rate was 97 $\mathrm{mm} / \mathrm{h}$; an urinalysis was normal. Serology for Epstein-Barr virus was negative. A Mantoux test placed on admission was negative when read at $48 \mathrm{~h}$. Ultrasound investigation of the neck mass excluded the possibility of an abscess. During hospitalization, he continued to have a fever of 38.5 to $38.8^{\circ} \mathrm{C}$. On the fifth day of admission, he was noted to have a rash on the flexor areas, around the large joints, and on his palms and soles. The site of a prior BCG vaccination was raised and erythematous (Figure 1).

What is the diagnosis?
\end{abstract}

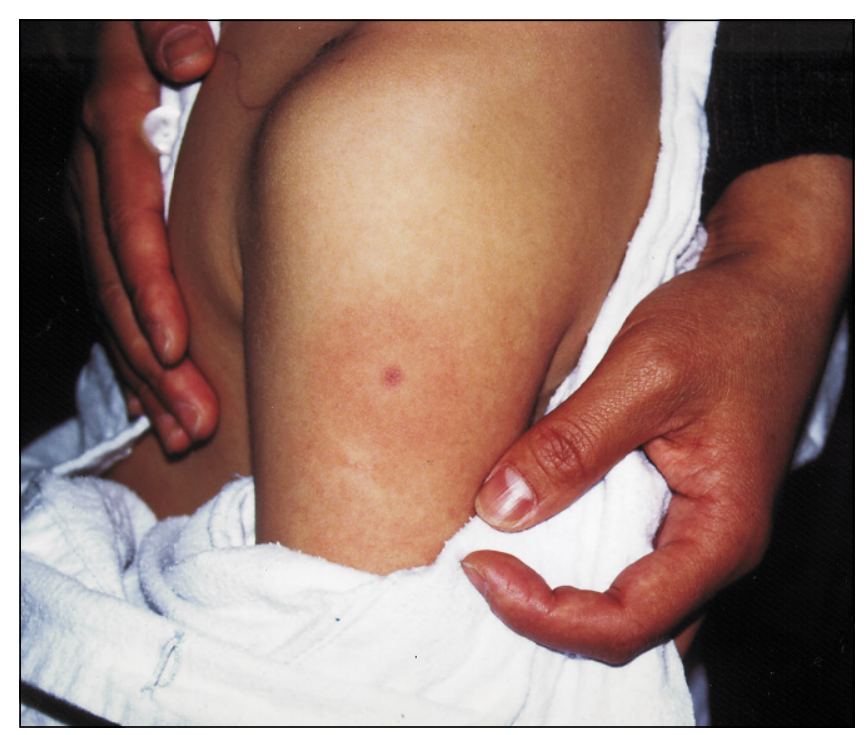

Figure 1) The site of previous bacillus Calmette-Guérin vaccination, as seen during the child's current febrile illness

\section{DIAGNOSIS}

The 'reactivation' of a BCG site in this patient is characteristic of Kawasaki disease (KD). The diagnosis was ultimately confirmed when the patient fulfilled the usual criteria for KD: fever for more than five days, bilateral conjunctivitis, cervical lymphadenopathy, generalized rash, erythema of the palms and soles, and subsequent development of erythematous oral mucosa. The patient received both intravenous immunoglobulin $(2 \mathrm{~g} / \mathrm{kg})$ and acetylsalicylic acid. As well, his neck swelling and irritability improved markedly. He was evaluated by a cardiologist and underwent an echocardiogram, which was normal. He was discharged on acetylsalicylic acid $(5 \mathrm{mg} / \mathrm{kg} /$ day $)$.

\footnotetext{
${ }^{1}$ Infectious Diseases Fellow and ${ }^{2}$ Division of Pediatric Rheumatology, BC's Children's Hospital, Vancouver, British Columbia

Correspondence and reprints: Dr David Cabral, 3321-4480 Oak Street, Vancouver, British Columbia V6H 3V4. Telephone 604-875-2134, fax 604-875-3649
} 


\section{DISCUSSION}

Erythema at the BCG site has been recognized as an early and specific manifestation of KD (1). The BCG site reactivation can vary from mild erythema and induration to ulceration. In cases where the BCG site has been examined specifically, Ziehl staining, culture and polymerase chain reaction studies for mycobacteria have been negative (2). The mechanism of this BCG reactivation is unknown. It is speculated that an antigen from the putative infectious agent(s) causing KD cross-reacts with the mycobacterial BCG antigens and thereby contributes to an inflammatory reaction. In Japan, where BCG is routinely administered to children, this phenomenon is widely recognized as an associated feature of KD (3). This phenomenon is less likely to be observed in North America, where BCG vaccination is uncommon.

Some patients who are suspected of having KD do not demonstrate the full diagnostic criteria; the finding of additional features known to be associated with KD supports the diagnosis of an 'incomplete' or 'atypical' KD. Noncardiac features associated with KD include extreme irritability, aseptic meningitis, pneumonitis, sterile pyuria, elevated liver transaminases, uveitis, otitis media, diarrhea, hydropic gall bladder and reactivation of a BCG site. BCG reactivation and hydrops of the gall bladder are relatively specific for $\mathrm{KD}$, and provide stronger support for 'atypical' KD than some of the other associated features.

\section{REFERENCES}

1 Yanagawa H, Nakamura Y, Kawasaki T, Shigematsu I. Nationwide epidemic of Kawasaki disease in Japan during winter of 1985-86. Lancet 1986;ii:1138-9.

2. Kuniyuki S, Asada M. An ulcerated lesion at the BCG vaccination site during the course of Kawasaki disease. J Am Acad Dermatol 1997;37:303-4.

3. Rowley AH, Shulman ST. Kawasaki syndrome. Pediatr Clin North Am 1999;46:313-29. 


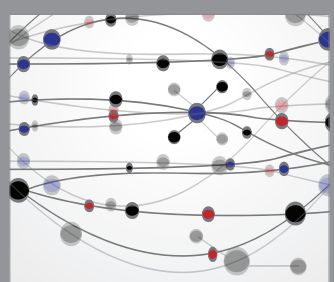

The Scientific World Journal
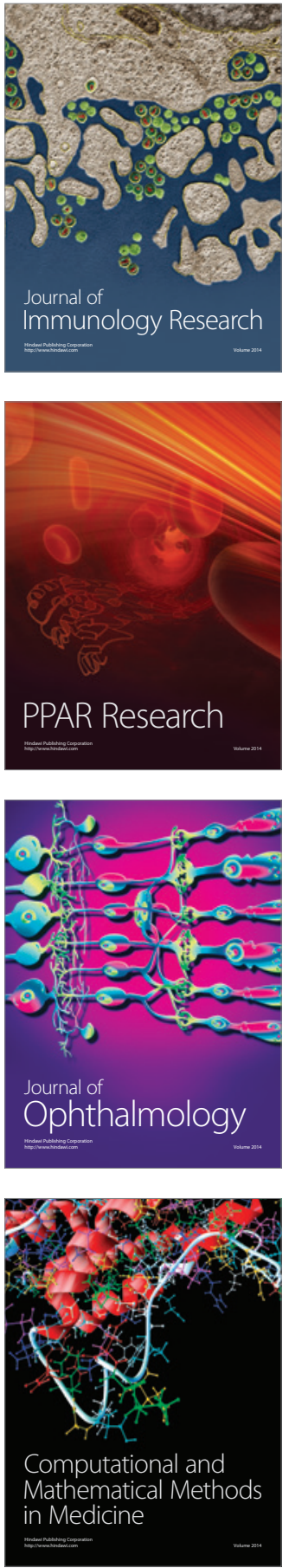

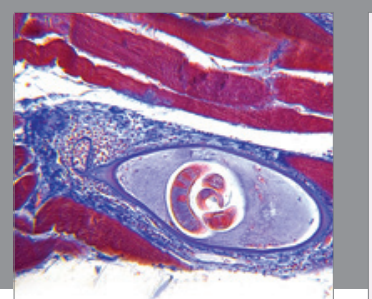

Gastroenterology Research and Practice

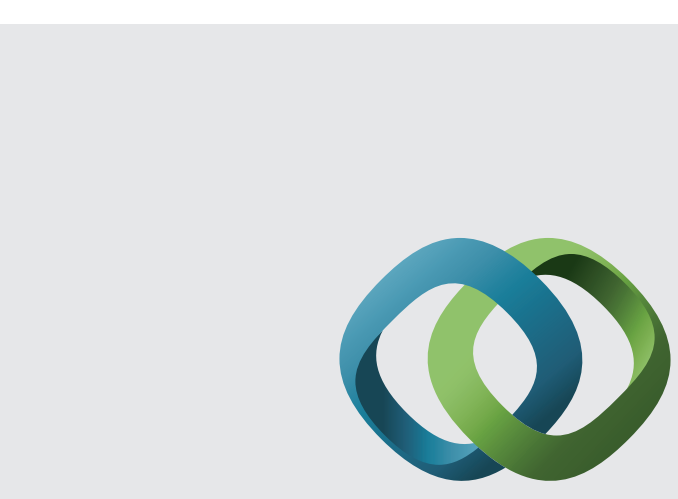

\section{Hindawi}

Submit your manuscripts at

http://www.hindawi.com
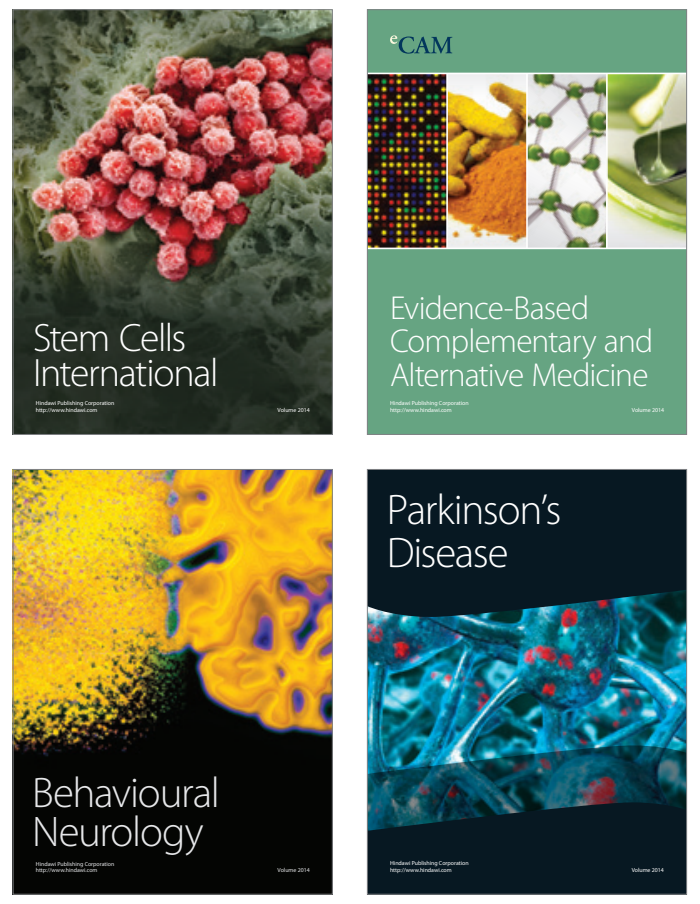
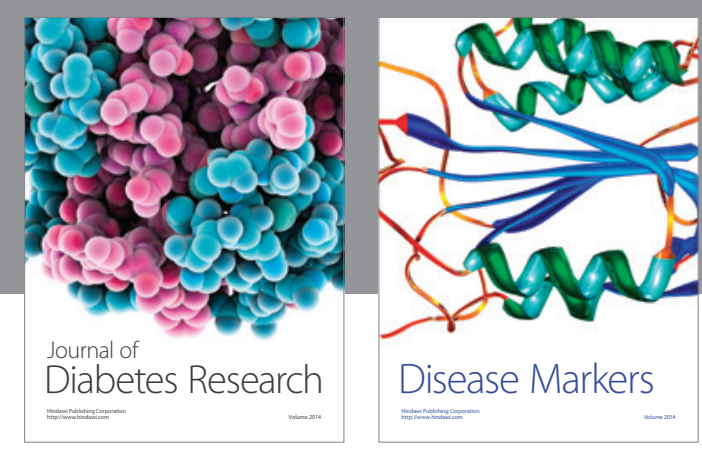

Disease Markers
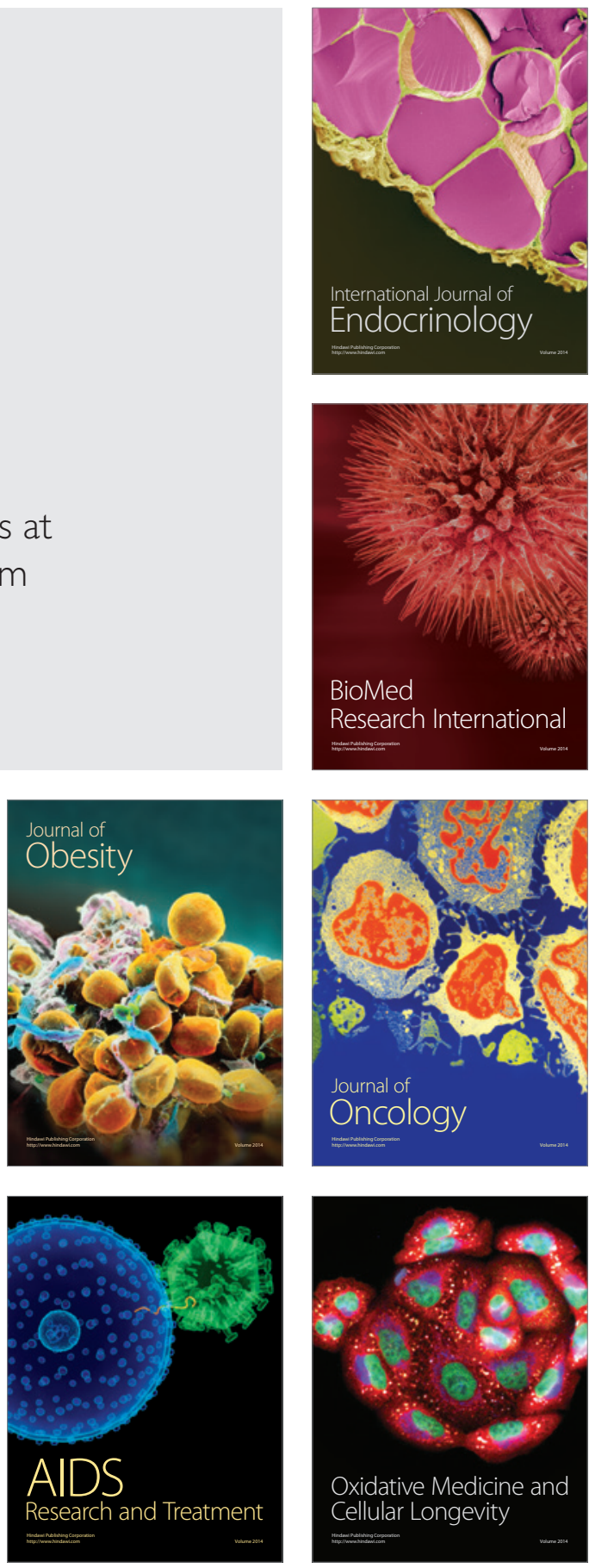